\title{
Analysis of Co-Channel Interference in Connected Vehicles WLAN with UAV
}

\author{
Maolin Wang, ${ }^{1,2} \mathrm{Xu}$ Ma $\mathbb{D}^{3},{ }^{3}$ Zhi Wang, ${ }^{1,2}$ and Yang Guo ${ }^{4}$ \\ ${ }^{1}$ Zhe Jiang Key Laboratory of General Aviation Operation Technology, Jiande, China \\ ${ }^{2}$ CAAC Key Laboratory of General Aviation Operation, Department of General Aviation, \\ Civil Aviation Management Institute of China, Beijing, China \\ ${ }^{3}$ University of Science and Technology Beijing, Beijing, China \\ ${ }^{4}$ Academy of Military Science of the PLA, Beijing 100000, China
}

Correspondence should be addressed to Xu Ma; 908033162@qq.com

Received 17 November 2021; Accepted 8 January 2022; Published 31 January 2022

Academic Editor: Renchao Xie

Copyright ( $) 2022$ Maolin Wang et al. This is an open access article distributed under the Creative Commons Attribution License, which permits unrestricted use, distribution, and reproduction in any medium, provided the original work is properly cited.

Unmanned aerial vehicle (UAV) has the advantages of flexibility, strong controllability, and easy deployment. It provides wireless communication with low delay and high throughput. However, under the background of the increasing shortage of spectrum resources, the frequency band resources of UAV systems are tight, resulting in increasingly serious co-channel interference between services, and the communication quality cannot be effectively guaranteed. Therefore, it is very important to evaluate the co-channel interference of UAV system and improve its spectral efficiency. This paper introduces the interference model for the research and analysis of the frequent coexistence of UAV and connected vehicles WLAN system and applies the model to evaluate the co-channel interference of UAV in the ISM frequency band of $2.4 \mathrm{GHz}$. The actual receiver filter is modeled and analyzed, the FDR mathematical expression for changing the protection band is derived, and two UAV interference signal waveforms, cyclic prefix orthogonal frequency division multiplexing waveform and windowed orthogonal frequency division multiplexing waveform, are proposed to effectively improve the utilization of frequency resources. After simulation, the interference of the interfered connected vehicles WLAN system is calculated and compared with the relevant service interference threshold standards issued by the International Telecommunication Union. To ensure that the UAV will not interfere with the connected vehicles WLAN system in the ISM frequency band of $2.4 \mathrm{GHz}$, the protection height of the UAV system in China is $6.2 \mathrm{~km}$.

\section{Introduction}

In recent years, the UAV communication system is popular in the field of wireless communication because of its flexibility, strong controllability, and easy deployment [1]. To lay the foundation for the era of "UAV Internet", UAV communication must support ubiquitous coverage, low delay, high reliability, and high throughput to realize the safe operation of UAV [2]. Compared with ground communication, since multipath and obstacles are mainly NLOS transmission, UAV communication can flexibly deploy UAVs according to the actual geographical location. Through LOS transmission, the path loss can be availably reduced, and the system communication performance can be extremely improved [3]. However, under the background of the increasing shortage of spectrum resources, the frequency band resources of UAV networks are tight, resulting in increasingly serious co-channel interference between services, and the communication quality cannot be effectively guaranteed [4]. In terms of the allocation and use of UAV spectrum resources, the contradiction between the frequency band selection and bandwidth demand of communication link and the shortage of spectrum resources is particularly prominent, which has become one of the important contents related to the healthy development of UAV industry [5].

Radio spectrum resources are owned by the state. China radio spectrum management and detection are mainly 
undertaken by the state radio regulation of China. To make rational use of resources, the MIIT has made plans for the use of radio spectrum. In 2015, the MIIT issued the notice of the frequency use of unmanned aerial vehicle systems, it specifies the spectrum range of communication links used for UAV systems, and stipulates that at present, Chinese UAV can only fly in isolated airspace, and its radio spectrum use shall comply with the national radio management regulations and shall not cause harmful interference to aviation radiofrequency. Most UAV data links and image transmission links adopt $2.4 \mathrm{GHz}$ industrial, scientific, and medical frequency bands (ISM) and use OFDM technology for signal transmission.

Table 1 shows the spectrum usage related to the UAV link planned by the MIIT of China. According to Chinese radio frequency division regulations,

840.5 to $845 \mathrm{MHz}$ and 2408 to $2440 \mathrm{MHz}$ frequency bands are shared with other existing services

429 to $1452 \mathrm{MHz}$ has been divided into fixed services, mobile services, and radio positioning services, but the 1430 to $1444 \mathrm{MHz}$ frequency band related to UAV planning spectrum is rarely occupied

Wireless local area network (WLAN) has been widely used due to its high transmission efficiency and good scalability [6]. Among them, the WLAN technology of the IEEE802.11 series has the most rapid development and is known as an effective scheme to solve the last $100 \mathrm{~m}$ access problem of the terminal $[7,8]$. The WLAN technology specified in IEEE802.11 b/g protocol [9] also works in the ISM frequency band. There may be spectrum interference between UAV and WLAN during use, which will impact their communication performance [10]. In the future, the intensive deployment of WLAN and the increase of communication bandwidth are bound to lead to more "congestion" of the ISM frequency band, and the same frequency interference will become more and more serious [11]. Therefore, evaluating the co-channel interference between UAV communication and connected vehicles WLAN system and improving its spectral efficiency has become a key problem.

Although UAV communication has attracted more and more attention in recent years, only a few works consider the problem of co-channel interference in connected vehicle WLAN with UAV. In Zhang et al.'s study [12], the interference-sensing path planning and design of UAV and ground base station under given communication quality of service requirements are studied. In Li et al.'s study [13], aiming at MITM attack, a communication resource optimal allocation scheme based on the A3C algorithm is proposed. In Van der Bergh et al.'s and Liu et al.'s studies $[14,15]$, the interference coordination in the uplink communication of $\mathrm{UAV}$ is designed, and the interference is regarded as noise to maximize the network throughput. However, the same frequency interference between UAV communication and the WLAN system is not considered in the aforementioned work. In Zhu et al.'s study [16], the proposed cross-layer defense scheme can effectively mitigate the jamming attack impact on CBTC. In Zhou et al.'s study [17], a mobile WLAN AP detection method based on noise application density spatial clustering (DBSCAN) is proposed for multidirectional positioning of UAV. In Topic et al.'s study [18], the basic characteristics of $2.4 \mathrm{GHz} \mathrm{UAV}$ and its behavior under interference are proposed. In Pandey et al.'s study [19], a multi-UAV flexible group task execution scheme based on WLAN is proposed. In Zhu et al.'s study [20], for connected vehicles, the problems of block producer selection and onboard blockchain client switching decisions are proposed.

Based on the aforementioned literature, this paper studies the co-channel interference between UAV communication and connected vehicles WLAN system based on the received SNR of the disturbed system. Its main contributions are summarized as follows:

Summarize China's current regulations on the use of UAV spectrum and the related radio spectrum division and use status

Aiming at the applicability of the shared spectrum between UAV and connected vehicles WLAN system, the interference scenario of UAV and satellite communication on the same frequency connected vehicles WLAN system is analyzed

Based on the interference scenario, interference estimation method and fixed parameters between UAV and connected vehicles WLAN, taking the SNR as the basis of interference analysis, calculate the interference of the interfered connected vehicles WLAN system, compare it with the relevant interference threshold standards issued by the ITU and determine the protection distance of UAV without affecting connected vehicles WLAN

The rest of this paper is organized as follows: Section 2 introduces the system model and proposes the interference estimation method. In Section 3, the fixed parameters of the UAV and connected vehicles WLAN system are determined and simulated, and the simulation results are given. Finally, the paper is concluded in Section 4 .

\section{System Model}

In this section, the interference model and interference estimation method in the coexistence research of UAV and connected vehicles WLAN systems are mainly introduced.

2.1. Interference Model. To make the UAV in the ISM band coexist with the connected vehicles WLAN system, it is necessary to analyze its interference. MCL method is adopted for interference analysis, which mainly solves the one-to-one situation, that is, the interference distributed by a single interference source system and victim system [21]. MCL minimizes the coupling loss between the UAV system and connected vehicles WLAN system, which is equivalent to assuming the maximum interference power. Through analysis, the equivalence of minimum separation distance and frequency interval can be determined [22-24]. 
TABLE 1: Spectrum use scope of UAV stipulated by the MIIT of China.

\begin{tabular}{lcc}
\hline Spectrum range $/ \mathrm{MHz}$ & Link usage & Other existing businesses \\
\hline $840.5-845$ & Uplink remote control link & RFID technology \\
$1430-1444$ & Downlink telemetry and information transmission link & Rarely occupied \\
$2408-2440$ & Uplink remote control, downlink telemetry, and information transmission link & WLAN technology \\
\hline
\end{tabular}

The interference model is based on the MCL method to simulate the real interference effect between UAV and connected vehicles WLAN. Figure 1 shows the interference model of connected vehicles WLAN receiver interfered by an UAV. It is assumed that the satellite, UAV, and the WLAN receiver of the interfered connected vehicle are on the same horizontal line, and they point to each other in the direction of the direction angle between the UAV and the interfered receiver. Considering that the antenna direction of the interfered receiver is relatively free, it is assumed to point directly to the UAV, and the element, as well as array gain is set to the maximum. For the frequency correlation suppression of the interfered receiver, to adapt to the channel interference, suppress out-of-band emission (OOBE), and reduce the interference of adjacent channels, two OFDM waveforms are used in UAV communication: cyclic prefix orthogonal frequency division multiplexing and windowed orthogonal frequency division multiplexing. Considering the actual receiver filter, the FDR of the actual receiver filter is modeled, and the mathematical expression of frequency correlation suppression of two OFDM waveforms in different guard bands is derived [25].

Suppose the UAV flies at a constant altitude and elevation, where $\theta_{\text {ele }}$ represents the elevation of the satellite, it is considered as the antenna elevation of the UAV. The interference path is the connection from the UAV to the disturbing service. To better understand the interference between UAV and connected vehicles WLAN jamming receiver, this paper considers the influence of four different UAV elevation angles on satellites $\left(5^{\circ}, 15^{\circ}, 30^{\circ}, 45^{\circ}\right)$ of UAV towards the satellite, the interference of each elevation angle is analyzed in ISM frequency band. The purpose of this analysis is to calculate the minimum separation distance from UAV to WLAN receiver in the worst case. In this case, in the horizontal domain, it is assumed that the connected vehicles WLAN receiver is satellite oriented and located in the main direction of the UAV. In the vertical domain, it is assumed that the antenna of the WLAN receiver is always facing the UAV.

\subsection{Interference Estimation Method}

2.2.1. SNR. SNR is the most commonly used index in interference analysis. In the interference scenario in Figure 1, the minimum separation distance between UAV and connected vehicles WLAN system is determined by calculating the interference noise ratio between UAV and interfered receiver at different distances and comparing it with the protection standard of connected vehicles WLAN, so as not to cause strong interference to connected vehicles WLAN. The ACI interference power can be expressed as follows:

$$
I_{A C I}=P_{\text {aero_ESIM }}-A_{\text {ESIM }}-P_{f s}-P_{\text {Fuselage }}-P_{\text {atmospheric }}+G_{r}-F D R,
$$

where $P_{\text {aero_UAV }}$ is e.i.r.p. of UAV, $A_{U A V}$ is the adjustment factor caused by bandwidth mismatch between UAV and WLAN, $P_{f s}$ is the free-space attenuation, $P_{\text {Fuselage }}$ is the fuselage loss of UAV, $P_{\text {atmospheric }}$ is the atmospheric gas loss, $G_{r}$ is the antenna gain of disturbed receiver, and FDR is the false discovery rate (frequency response ratio).

As the interference power exceeds a certain value, the ACI from the UAV leads to the interruption of the interfered system. Therefore, the interference power of the UAV needs to be within the acceptable range of the interfered system. The maximum acceptable interference of the interfered system can be calculated from the signal-to-noise ratio threshold, shown as follows:

$$
I_{t h}=\frac{I}{N_{t h}+N_{\mathrm{Nol}}} .
$$

where $N_{N o l}$ is the total power of the interfered WLAN (considering thermal noise and noise coefficient). Different maximum acceptable interference needs to be determined according to the signal-to-noise ratio threshold of different services. For $N_{N o l}$, it is mainly related to the temperature, bandwidth, and noise coefficient of the disturbed system, shown as follows:

$$
N_{N o l}=10 \log (K T)+N F+10 \log \left(B W_{\text {victim }}\right) .
$$

$B W_{\text {victim }}$ is bandwidth of the disturbed system.

2.2.2. Equivalent Isotropically Radiated Power. According to relevant resolutions and standards issued by WRC-19 and referring to ITU-R S.524-9 [26], the off-axis-equivalent isotropically radiated power $P_{\text {aero_UAV }}$ is the sum of output 


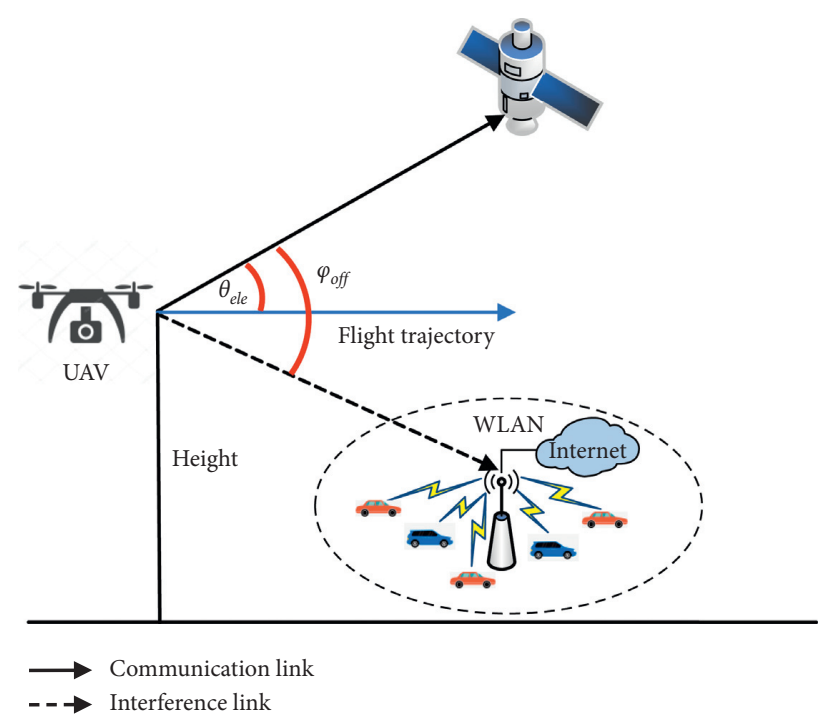

FIGURE 1: Co-channel interference model between UAV and connected vehicles WLAN.

power and antenna gain when the off-axis angle is $\phi_{o f f}$, which can be expressed as follows:

$$
P_{\text {aero } \_U A V}\left(\phi_{o f f}\right)=\left\{\begin{array}{l}
19-25 \log _{10} \phi_{o f f}, 2^{\circ} \leq \phi_{o f f} \leq 7^{\circ} \\
-2,7^{\circ}<\phi_{o f f} \leq 9.2^{\circ} \\
22-25 \log _{10} \phi_{o f f}, 9.2^{\circ}<\phi_{o f f} \leq 48^{\circ} \\
-10,48^{\circ}<\phi_{o f f} \leq 180^{\circ}
\end{array} .\right.
$$

2.2.3. Free-Space Loss. For the convenience of analysis, the link loss between UAV and interfered receiver adopts the free-space loss model, referring to ITU-R P.525 [27], which is specifically expressed as follows:

$$
P_{f s}=92.4+20 \log _{10} f+20 \log _{10} d \text {. }
$$

2.2.4. Fuselage Loss. For the fuselage loss of UAV, the signal attenuation occurs in the UAV fuselage. Refer to the model in ITU-R M.2221 [28], and the specific relationship between fuselage loss and off-axis angle is shown in Figure 2. Based on the MCL method, assuming the worst interference, the model can be applied to this paper.

2.2.5. Atmospheric Gas Loss. The downward path gas attenuation of UAV and connected vehicles WLAN can be calculated by line-by-line method.

The gas attenuation of the downward inclined path between heights $h_{1}$ and $h_{2}\left(h_{2}>h_{1} \geq 0 \mathrm{~km}\right)$ is as follows:

$$
\begin{aligned}
P_{\text {gas }} & =\int_{h_{1}}^{h_{2}} \frac{\gamma(h)}{\sin \phi(h)} d h=\int_{h_{1}}^{h_{2}} \frac{\gamma(h)}{\sqrt{1-\cos ^{2} \phi(h)}} d h, \\
\cos \phi(h) & =\frac{\left(R_{E}+h_{1}\right) n\left(h_{1}\right)}{\left(R_{E}+h\right) n(h)} \cos \phi_{1}, \\
\gamma & =\gamma_{o}+\gamma_{w}=0.1820 f\left(N_{\text {Oxygen }}^{\prime \prime}(f)+N_{\text {WaterVapour }}^{\prime \prime}(f)\right),
\end{aligned}
$$

where $\gamma_{o}$ and $\gamma_{w}$ are the specific attenuation under dry air (nitrogen and nonresonant Debye attenuation caused by air pressure under oxygen condition) and water vapor condition, respectively. $f$ is the frequency, and $N_{\text {Oxygen }}^{\prime \prime}(f)$ and $N_{\text {WaterVapour }}^{\prime \prime}(f)$ are imaginary parts of the negative refractive index associated with that frequency. 


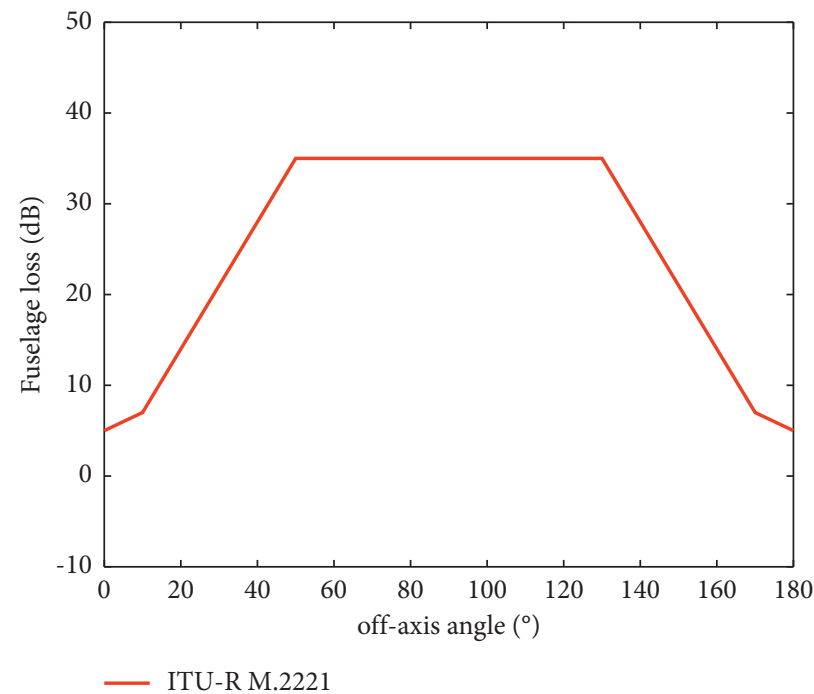

FIGURE 2: Relationship between fuselage loss and off-axis angle.

$$
\begin{aligned}
N_{\text {Oxygen }}^{\prime \prime}(f) & =\sum_{i(\text { Oxygen })} S_{i} F_{i}+N_{D}^{\prime \prime}(f), \\
N_{\text {WaterVapour }}^{\prime \prime}(f) & =\sum_{i \text { (WaterVapour })} S_{i} F_{i},
\end{aligned}
$$

$S_{i}$ is the intensity of oxygen or water vapor spectral line in article $i$, and $F_{i}$ is the shape factor of oxygen or water vapor spectral line. The data can refer to any of the six reference standard atmospheres provided in ITU-R P.676 [29], In this paper, the global average reference atmospheric data are used, and $a, b$, and $f_{0}$ in the following are the spectral line data.

The spectral line intensity is as follows:

$$
\begin{aligned}
& S_{i}=a_{1} \times 10^{-7} P \theta^{3} \exp \left[a_{2}(1-\theta)\right] \text { for oxygen } \\
& S_{i}=b_{1} \times 10^{-1} e \theta^{3.5} \exp \left[b_{2}(1-\theta)\right] \text { for watervapour, }
\end{aligned}
$$

where $P$ is the dry air pressure $(h P a), e$ is the partial pressure of water vapor $(h P a)$, and the total pressure is $P_{t o l}=P+e$; $\theta=300 / T$.

The spectral line shape factor is computed as follows:

$$
F_{i}=\frac{f}{f_{i}}\left[\frac{\Delta f-\delta\left(f_{i}-f\right)}{\left(f_{i}-f\right)^{2}+\Delta f^{2}}+\frac{\Delta f-\delta\left(f_{i}+f\right)}{\left(f_{i}+f\right)^{2}+\Delta f^{2}}\right],
$$

where $f_{i}$ is the spectral line frequency of oxygen or water vapor, and $\Delta f$ is the spectral line width:

$$
\begin{aligned}
& \Delta f=a_{3} \times 10^{-4}\left(\rho \theta^{\left(0.8-a_{4}\right)}+1.1 e \theta\right) \text { for oxygen, } \\
& \Delta f=b_{3} \times 10^{-4}\left(\rho \theta^{b_{4}}+b_{5} e \theta^{b_{6}}\right) \text { for water vapor. }
\end{aligned}
$$

Modify the spectral line width $\Delta f$ :

$$
\begin{aligned}
\Delta f= & \sqrt{\Delta f^{2}+2.25 \times 10^{-6}} \text { for oxygen, } \\
\Delta f= & 0.535 \Delta f \\
& +\sqrt{0.217 \Delta f^{2}+\frac{2.1316 \times 10^{-12} f_{i}^{2}}{\theta}} \text { for water vapor. }
\end{aligned}
$$

Due to the interference in the oxygen line and $\delta$ is the correction factor. For oxygen, $\delta=\left(a_{5}+a_{6} \theta\right) \times 10^{-4}(p+e) \theta^{0.8}$. For water vapor, $\delta=0$.

$N_{D}^{\prime \prime}(f)=f p \theta^{2}\left[\frac{6.14 \times 10^{-5}}{d\left[1+(f / d)^{2}\right]}+\frac{1.4 \times 10^{-12} p \theta^{1.5}}{1+1.9 \times 10^{-5} f^{1.5}}\right]$.

2.2.6. False Discovery Rate. In the study of frequency sharing, false discovery rate (FDR) can be calculated as follows [30]:

$$
F D R=10 \log _{10}\left(\frac{\int_{-\infty}^{\infty} \Phi(f) d f}{\int_{-\infty}^{\infty} \Phi(f) \Psi(f-\Delta f) d f}\right),
$$

where $\Phi(f)$ is the PSD of the interference signal, $\Psi(f)$ is the frequency response of the receiver, and $\Delta f$ is the frequency offset. The guard band can be calculated by frequency offset:

$$
G B=\Delta f-\frac{W_{T}}{2}-\frac{W_{R}}{2} .
$$

In this paper, two OFDM signals are used to calculate FDR, namely cyclic prefix OFDM and windowed OFDM. The actual frequency response is obtained through receiver modeling, and the FDR is calculated according to the signal. The specific expression is as follows: 


$$
\Psi(f)[d B]=\left\{\begin{array}{l}
b,|f| \geq \frac{W_{t}}{2}, \\
0,|f| \leq \frac{W_{f}}{2}, \\
\left(\frac{2 b}{\left(W_{f}-W_{t}\right)}\right)\left(f+\frac{W_{f}}{2}\right),-\frac{W_{t}}{2}<f<-\frac{W_{f}}{2}, \\
\left(\frac{2 b}{\left(W_{t}-W_{f}\right)}\right)\left(f-\frac{W_{f}}{2}\right), \frac{W_{f}}{2}<f<\frac{W_{t}}{2},
\end{array}\right.
$$

where $W_{f}$ is the flat response bandwidth, $W_{t}$ is the conversion bandwidth, and $W_{R}$ is the receiver channel bandwidth.

To solve the problem of interchannel interference, the cyclic prefix is executed by placing the protection interval between data symbols, which is called cyclic prefix orthogonal frequency division multiplexing, and its baseband signal is represented as follows:

$$
s(t)=\sum_{n=-\infty}^{\infty} \sum_{k=0}^{N-1} c_{n, k} p\left(t-n\left(T_{S}+T_{g}\right)\right) e^{-j 2 \pi k 1 / N\left(T_{s}+T_{g}\right)}
$$

The power spectral density of the orthogonal frequency division multiplexing interference signal using any pulseshaping window can be expressed as follows:

$$
\Phi(f)=\frac{P_{s}}{T_{\text {total }}} \sum_{k=0}^{N-1}\left|P\left(f-\frac{k}{T_{s}}\right)\right|^{2}
$$

The cyclic prefix OFDM subcarrier is represented as follows:

$$
p_{c p}(t)=\Pi\left(\frac{t}{T_{\text {total }}}\right)= \begin{cases}0, & t>\left|\frac{T_{\text {total }}}{2}\right|, \\ 1, & t \leq\left|\frac{T_{\text {total }}}{2}\right| .\end{cases}
$$

The Fourier transform of the aforementioned formula can be expressed as follows:

$$
P_{C P}(f)=T_{\text {total }} \cdot \sin c\left(T_{\text {total }} f\right),
$$

where $\sin c(x)=\sin (x \pi) / x \pi$. Substituting formula (25) into (23) can obtain the power spectral density of cyclic prefix OFDM:

$$
\Phi_{C P}(f)=P_{s} T_{\text {total }} \sum_{k=0}^{N-1}\left|\sin c\left[\left(f-\frac{k}{T_{s}}\right) T_{\text {total }}\right]\right|^{2} .
$$

For windowed OFDM, the window of the raised cosine function is as follows:

$$
p_{w o}(t)= \begin{cases}1, & |t| \leq \frac{\left(T_{\text {total }}-T_{t r}\right)}{2} \\ \frac{1}{2}\left(1+\cos \left(\frac{\pi\left(|t|-\left(T_{\text {total }}-T_{t r}\right) / 2\right)}{T_{t r}}\right)\right) & \frac{\left(T_{\text {total }}-T_{t r}\right)}{2} \leq|t|<\frac{T_{w}}{2}, \\ 0, & \frac{T_{w}}{2} \leq|t|,\end{cases}
$$


where $T_{t r}$ is the transition time and $T=T_{\text {total }}+T_{t r}$ is the window duration. The Fourier transform of formula (27) is as follows:

$$
P_{W O}(f)=\frac{T_{\text {total }} \sin c\left(T_{\text {total }} f\right) \cdot \cos \left(\pi T_{t r} f\right)}{1-4 T_{t r}^{2} f^{2}} .
$$

Substitute formula (28) into (23) to obtain the power spectral density of windowed orthogonal frequency division multiplexing:

$$
\Phi_{W O}(f)=P_{s} T_{\text {total }} \sum_{k=0}^{N-1}\left\{\sin c\left[\left(f-\frac{k}{T_{s}}\right) T_{\text {total }}\right] \times \frac{\cos \left(\pi T_{t r}\left(f-k / T_{s}\right)\right)}{1-4 T_{t r}^{2}\left(f-k / T_{s}\right)^{2}}\right\}^{2}
$$

The power spectral density and formula (25) of the obtained orthogonal frequency division multiplexing waveform are substituted into formula (23), and the frequency response ratio is obtained by the following formula:

$$
\begin{aligned}
F D R & =P_{t}-10 \log _{10}\left(\int_{-\infty}^{\infty} \Phi(f) \Psi(f-\Delta f)\right) \\
& =P_{t}-10 \log _{10}\left(\int_{-\infty}^{\Delta f-W_{t} / 2} \Phi(f) \cdot 10^{b / 10} d f+\int_{\Delta f-W_{t} / 2}^{\Delta f-W_{v} / 2} \Phi(f) \cdot 10^{\left(\left(2 b / W_{v}-W_{s}\right)\left(f-\Delta f+W_{v} / 2\right)\right)} d f\right. \\
& \left.+\int_{\Delta f-W_{v} / 2}^{\Delta f+W_{v} / 2} \Phi(f) d f+\int_{\Delta f+W_{v} / 2}^{\Delta f+W_{t} / 2} \Phi(f) \cdot 10^{\left(\left(2 b / W_{t}-W_{v}\right)\left(f-\Delta f-W_{v} / 2\right)\right)} d f+\int_{\Delta f+\frac{W_{t}}{2}}^{\infty} \Phi(f) \cdot 10^{b / 10} d f\right)
\end{aligned}
$$

where $P_{t}=\int_{-\infty}^{\infty} \Phi(f) d f$.

\section{System Simulation}

In this section, we focus on the relevant parameters of cochannel interference analysis of UAV and connected vehicles WLAN system, as well as the receiver filter and interference signal parameters when calculating false discovery rate. Then, we analyze the simulation results of the same frequency interference model.

3.1. Parameter Setting. The main parameters of the UAV system can be determined according to the relevant recommendations of ITU-R, as shown in Table 2.

The system parameters of WLAN used for interference evaluation are shown in Table 3.

When calculating the false discovery rate, the parameters of receiver filter and orthogonal frequency division multiplexing parameters of interference signal shall be considered, as shown in Tables 4 and 5.

3.2. Analysis of Simulation Results. In the aforementioned interference scenario between UAV and connected vehicles WLAN service, the interference level is calculated considering the mobility of UAV. To consider various factors that may affect the interference level, the receiver filters of different positions, elevations, and disturbed systems are selected for analysis in the simulation. According to the system parameters, the same frequency interference between UAV and connected vehicles WLAN is analyzed. In the ISM band, the relationship between atmospheric gas attenuation of the interference signal and separation distance between UAV and connected vehicles WLAN service is shown in Figure 3. The higher the UAV height, the greater the atmospheric gas attenuation.

In this paper, the interference of UAV to connected vehicles WLAN system at different elevation angles, $2.4 \mathrm{GHz}$, zero protection bandwidth and different altitudes is considered. Figures 4(a), 4(b), 5(a), and 5(b), respectively, show the I/N of UAV and connected vehicles WLAN interference path at different heights when the elevation angle of UAV is $5^{\circ}, 15^{\circ}, 30^{\circ}$, and $45^{\circ}$ by MCL method. As can be seen from the figure: the larger the elevation angle of UAV, the smaller the interference level to the connected vehicles WLAN system in ISM band, and the smaller the probability of co-channel interference between UAV and connected vehicles WLAN system. Figures 6(a) and 6(b) show the I/N of the interference path between UAV and connected vehicles WLAN when the separation height between UAV and connected vehicles WLAN system is $1 \mathrm{~km}$ and $6.2 \mathrm{~km}$. As can be seen from the figure: the greater the separation height between UAV and connected vehicles WLAN system, the smaller the interference level to connected vehicles WLAN system in ISM band, and the smaller the probability of cochannel between UAV and connected vehicles WLAN system. When the separation height between UAV and connected vehicles WLAN system is $6.2 \mathrm{~km}$, the elevation 
TABLE 2: Main parameters of the UAV system.

\begin{tabular}{lccc}
\hline Parameter & Unit & Value & Attention \\
\hline Frequency & $\mathrm{GHz}$ & $2.4-2.4835$ & ITU-R S.524 \\
e.i.r.p. & $d B \mathrm{~W} / 40 \mathrm{kHz}$ & Reference 2-2 & $5,15,30,45$ \\
UAV elevation & Degrees & 100 & \\
Bandwidth & $\mathrm{MHz}$ & & \\
\hline
\end{tabular}

TABLE 3: System parameters of WLAN for interference evaluation.

\begin{tabular}{|c|c|c|c|}
\hline Parameter & WLAN & Unit & Attention \\
\hline & Interfered receiver & & \\
\hline Frequency & $2.4-2.4835$ & $\mathrm{GHz}$ & \\
\hline Bandwidth & 20 & $\mathrm{MHz}$ & ITU-R S.524 \\
\hline Antenna height & 10 & meter & \\
\hline Polarization mode & Horizontal polarization vertical polarization & $d B i$ & \\
\hline Antenna pointing & Pointing to maximum gain & & \\
\hline Maximum gain & 19 & $d B$ & \\
\hline Noise figure & 10 & $d B$ & \\
\hline Protection standard & 25 & $d B$ & \\
\hline
\end{tabular}

TABle 4: Parameters of the receiver filter.

\begin{tabular}{lccc}
\hline Parameter & Value & Symbol & Unit \\
\hline Channel bandwidth & 100 & $W_{R}$ & $\mathrm{MHz}$ \\
Flat response bandwidth & $0.97 W_{R}$ & $W_{f}$ & $\mathrm{MHz}$ \\
Transition bandwidth & $1.97 W_{R}$ & $W_{t}$ & $\mathrm{MHz}$ \\
Stopband attenuation & -100 & $b$ & $d B$ \\
\hline
\end{tabular}

TABle 5: Parameters of the OFDM signal.

\begin{tabular}{lccc}
\hline Parameter & Value & Symbol & Unit \\
\hline Channel bandwidth & 20 & N/A & $\mathrm{MHz}$ \\
Signal bandwidth & 17.94 & $\mathrm{~N} / \mathrm{A}$ & $\mathrm{MHz}$ \\
Output power & 37 & $P_{t}$ & $d B \mathrm{~m}$ \\
Number of subcarriers & 672 & $\mathrm{~N}$ & $\mathrm{~N} / \mathrm{A}$ \\
Data duration & 8.33 & $T_{s}$ & $\mu s$ \\
Carrier spacing & 120 & $1 / T_{s}$ & $\mathrm{kHz}$ \\
Protection interval & 0.57 & $T_{g}$ & $\mu s$ \\
Transition time & 0.8228 & $T_{t r}$ & $\mu s$ \\
\hline
\end{tabular}

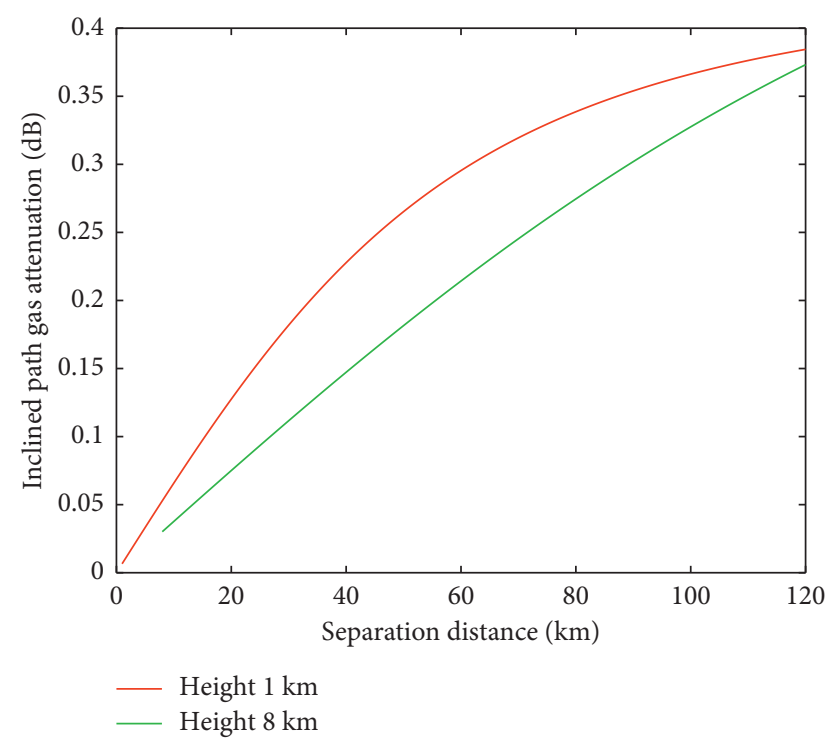

FIGURE 3: Relationship between atmospheric gas attenuation and separation distance in inclined path. 


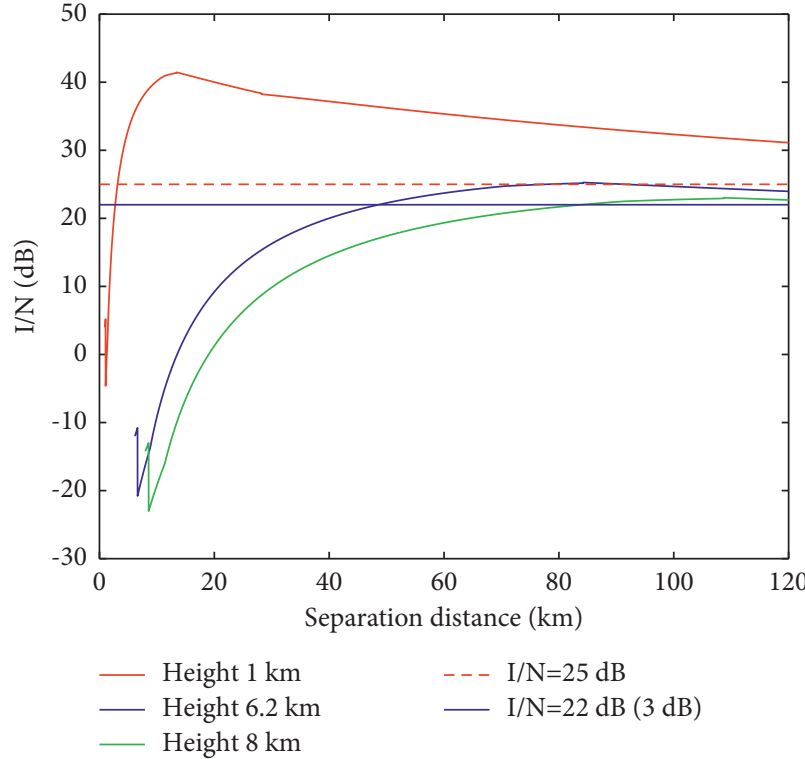

(a)

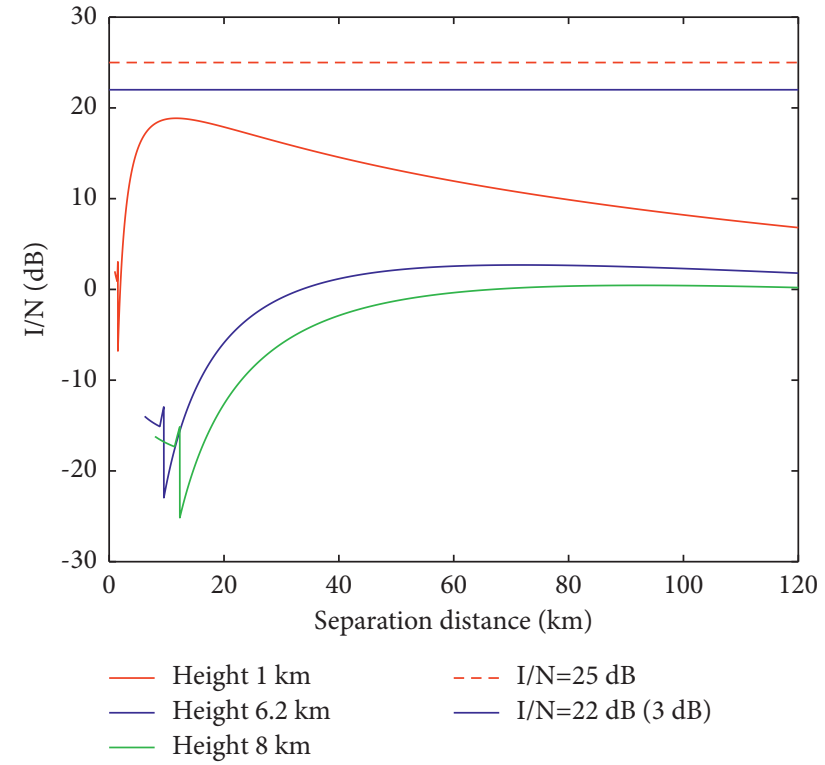

(b)

Figure 4: (a) I/N (interference to WLAN when elevation is $5^{\circ}$ ) and (b) I/N (interference to WLAN when elevation is $15^{\circ}$ ).

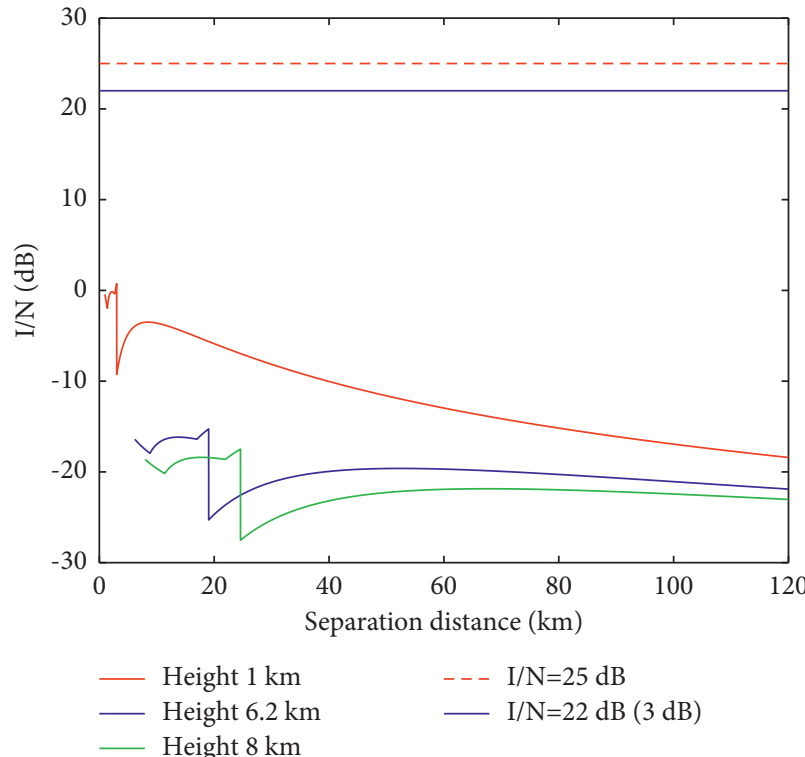

(a)

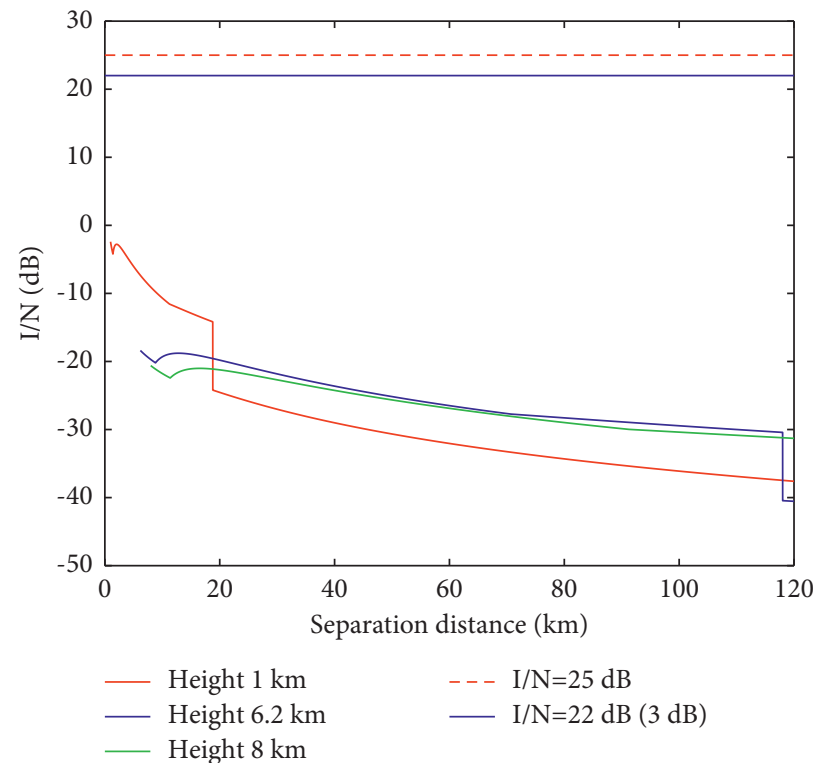

(b)

Figure 5: (a) I/N (interference to WLAN when elevation is $30^{\circ}$ ) and (b) I/N (interference to WLAN when elevation is $45^{\circ}$ ).

angle of UAV can ensure that there will be no co-channel interference to connected vehicles WLAN system under all circumstances. The required protection height between UAV and connected vehicles WLAN system is about $6.2 \mathrm{~km}$, respectively. At the same time, increasing the elevation of UAV or increasing the protection height can reduce the interference of UAV to connected vehicles WLAN system.

To draw a more intuitive conclusion, the cumulative distribution function of the interference signal-to-noise ratio under the aforementioned conditions is obtained, and the interference threshold standard is compared and analyzed. As shown in Figure 7(a), when the protection height is $6.2 \mathrm{~km}$ and the elevation angle of $\mathrm{UAV}$ is $5^{\circ}$, the interference level between UAV and connected vehicles WLAN system just reaches the same frequency interference threshold of connected vehicles WLAN system. When the height is greater than $6.2 \mathrm{~km}$, the interference level is lower than the interference threshold standard of connected vehicles 


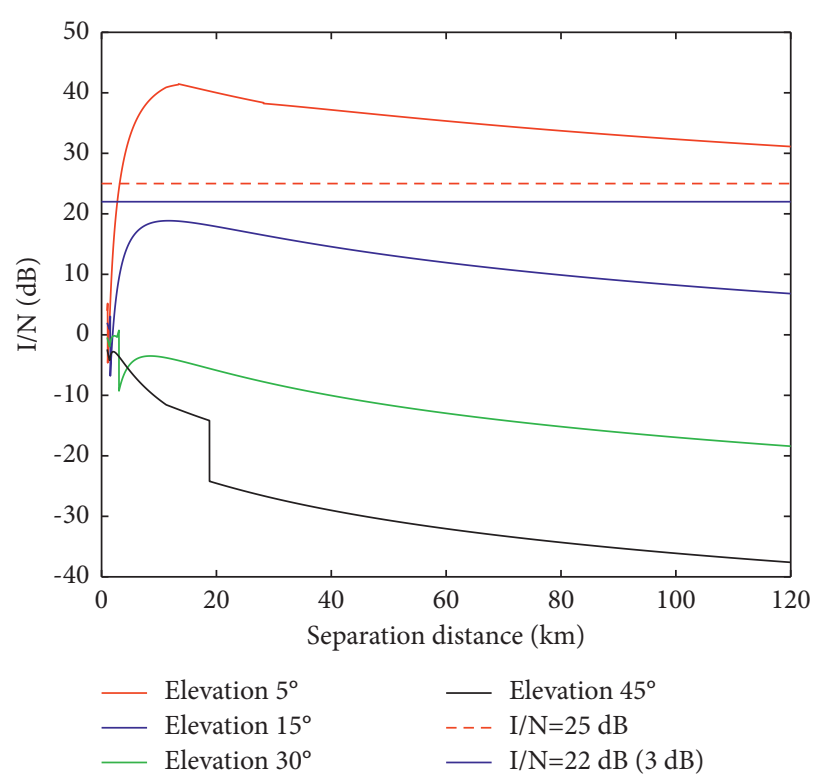

(a)

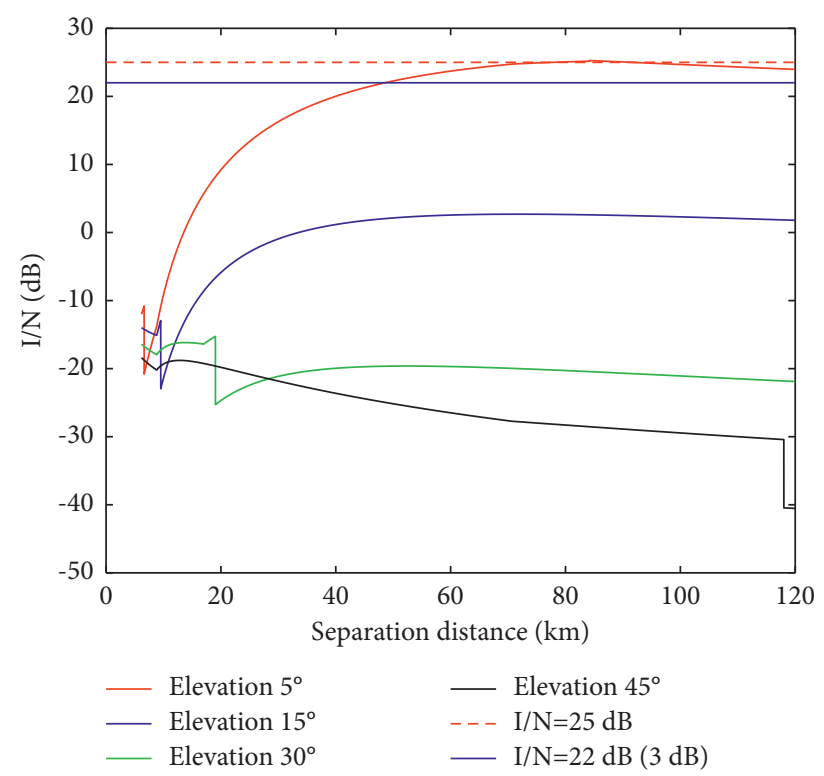

(b)

FIgURE 6: (a) I/N (interference to WLAN when height is $1 \mathrm{~km}$ ) and (b) I/N (interference to WLAN when height is $6.2 \mathrm{~km}$ ).

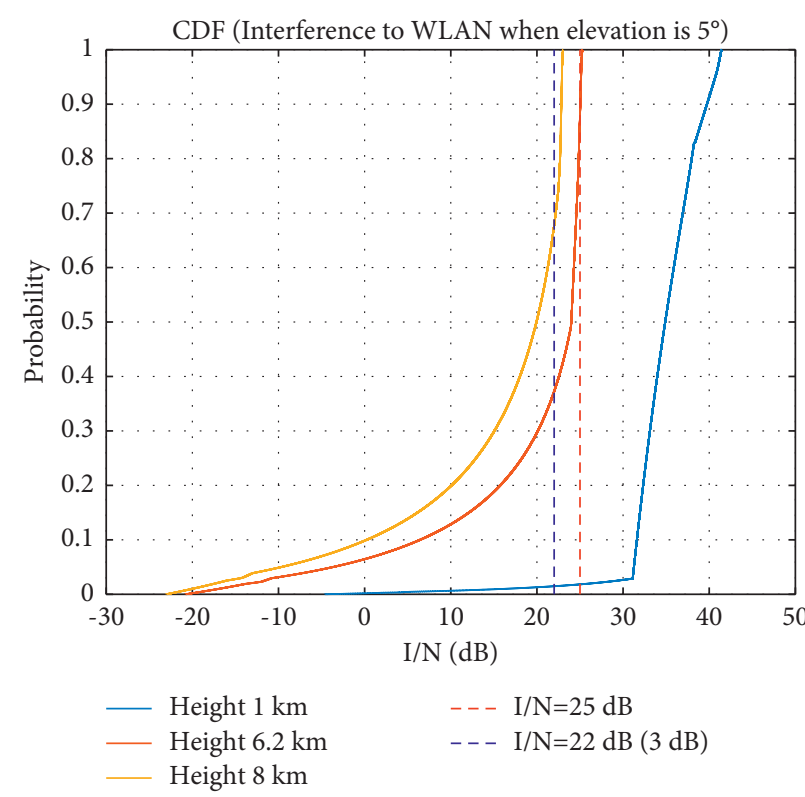

(a)

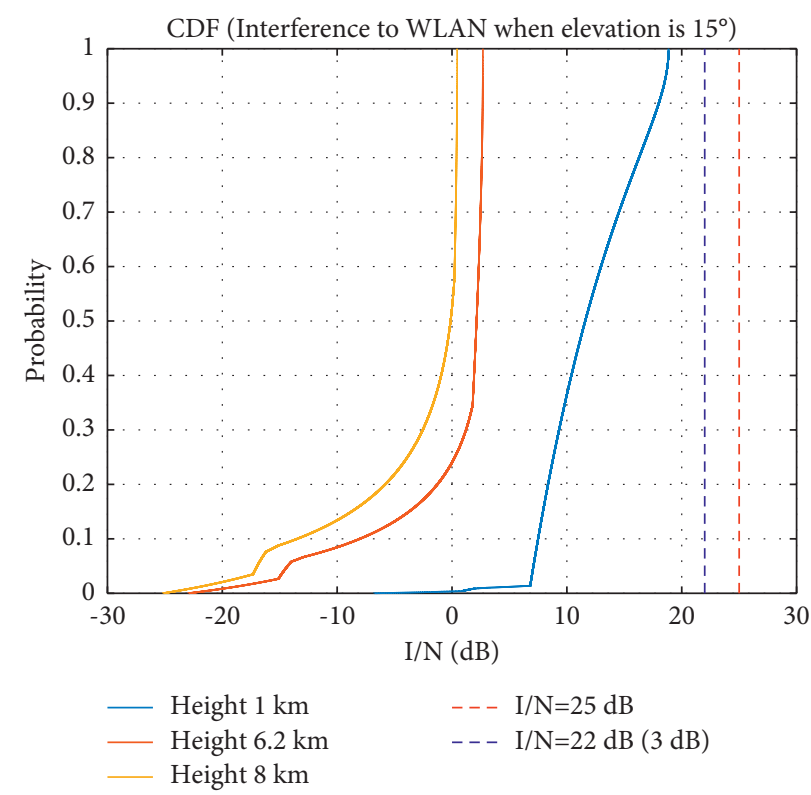

(b)

Figure 7: (a) CDF (interference to WLAN when elevation is $5^{\circ}$ ) and (b) I/N (interference to WLAN when elevation is $15^{\circ}$ ).

WLAN system. Figure 7(b) shows that when the elevation angle of UAV is $15^{\circ}, 30^{\circ}$, and $45^{\circ}$, and the height is greater than $1 \mathrm{~km}$, the interference level between UAV and connected vehicles WLAN system is lower than the interference threshold of the same frequency, and UAV will not interfere with WLAN system of the same frequency during operation under these conditions.

\section{Conclusion}

In this paper, we considered the co-channel interference in connected vehicles WLAN with UAV, analyzed the interference scene generated by UAV and satellite communication on the co-channel connected vehicles WLAN system, propose the UAV co-channel interference model, and apply 
the model to evaluate the interference between UAV and connected vehicles WLAN system in ISM frequency band. The simulation results show that when the separation height between UAV and connected vehicles WLAN system is not less than $6.2 \mathrm{~km}$, the elevation angle of UAV can ensure that there is no co-channel interference with connected vehicles WLAN system in all cases, and increasing the elevation angle or protection height of UAV can reduce the interference of UAV to connected vehicles WLAN system.

\section{Data Availability}

The data used to support the findings of this study are included within the article.

\section{Conflicts of Interest}

The authors declare that they have no conflicts of interest.

\section{Acknowledgments}

This work was supported by the National Natural Science Foundation of China (grant nos. U1933130, 71731001, 1433203, and U1533119), Zhe Jiang Key Laboratory of General Aviation Operation Technology (General Aviation Institute of Zhejiang JianDe) (no. JDGA2020-5), and Research Project of Chinese Academy of Sciences (grant no. ZDRW-KT-2020-21-2).

\section{References}

[1] Y. Zeng, Q. Wu, and R. Zhang, "Accessing from the sky: a tutorial on UAV communications for 5G and beyond," Proceedings of the IEEE, vol. 107, no. 12, pp. 2327-2375, 2019.

[2] P. H. Kopardekar, "Unmanned aerial system (UAS) traffic management (UTM): enabling low-altitude airspace and UAS operations," 2014.

[3] C. You and R. Zhang, "Hybrid offline-online design for UAVenabled data harvesting in probabilistic LoS channels," IEEE Transactions on Wireless Communications, vol. 19, no. 6, pp. 3753-3768, 2020.

[4] V. U. Pai and B. Sainath, "UAV selection and link switching policy for hybrid tethered UAV-assisted communication," IEEE Communications Letters, vol. 25, no. 7, pp. 2410-2414, 2021.

[5] T. Alladi, Naren, G. Bansal, V. Chamola, and M. Guizani, "SecAuthUAV: a novel authentication scheme for UAVground station and UAV-UAV communication," IEEE Transactions on Vehicular Technology, vol. 69, no. 12, pp. 15068-15077, 2020.

[6] P. Zhou, X. Fang, and X. Wang, "Joint radio resource allocation for decoupled control and data planes in densely deployed coordinated WLANs," IEEE Transactions on Wireless Communications, vol. 20, no. 6, pp. 3749-3759, 2021.

[7] M. Abusubaih, "Using partially overlapping channels in home 802.11g WLANs," Wireless Personal Communications, vol. 88, no. 2, pp. 295-303, 2016.

[8] Q. Chen and Y.-H. Zhu, "Scheduling channel access based on target wake time mechanism in 802.11ax WLANs," IEEE Transactions on Wireless Communications, vol. 20, no. 3, pp. 1529-1543, 2021.
[9] B. Bellalta, "Throughput analysis in high density WLANs," IEEE Communications Letters, vol. 21, no. 3, pp. 592-595, 2017.

[10] D. Chen, "Coexistence and interference mitigation for WPANs and WLANs from traditional approaches to deep learning: a Review," IEEE Sensors Journal, vol. 21, no. 22, pp. 25561-25589, 2021.

[11] S. Aust, R. V. Prasad, and I. G. M. M. Niemegeers, "Outdoor long-Range WLANs: a lesson for IEEE 802.11ah," IEEE Communications Surveys \& Tutorials, vol. 17, no. 3, pp. 1761-1775, 2015.

[12] S. Zhang, Y. Zeng, and R. Zhang, "Cellular-Enabled UAV communication: a connectivity-constrained trajectory optimization perspective," IEEE Transactions on Communications, vol. 67, no. 3, pp. 2580-2604, 2019.

[13] Y. Li, L. Zhu, H. Wang, F. R. Yu, and S. Liu, "A cross-Layer defense scheme for edge intelligence-enabled CBTC systems against MITM attacks," IEEE Transactions on Intelligent Transportation Systems, vol. 22, no. 4, pp. 2286-2298, 2021.

[14] B. Van der Bergh, A. Chiumento, and S. Pollin, "LTE in the sky: trading off propagation benefits with interference costs for aerial nodes," IEEE Communications Magazine, vol. 54, no. 5, pp. 44-50, 2016.

[15] L. Liu, S. Zhang, and R. Zhang, "Multi-Beam UAV communication in cellular uplink: cooperative interference cancellation and sum-rate maximization," IEEE Transactions on Wireless Communications, vol. 18, no. 10, pp. 4679-4691, Oct. 2019.

[16] L. Zhu, H. Liang, H. Wang, B. Ning, and T. Tang, "Joint security and train control design in blockchain empowered CBTC system," IEEE Internet of Things Journal, p. 1, 2021.

[17] M. Zhou, H. Yuan, Y. Wang, W. Tan, and Z. Tian, "Indoor UAV localization using manifold alignment with mobile ap detection," in Proceedings of the 2019 IEEE International Conference on Communications (ICC), pp. 1-6, Shanghai, China, 2019.

[18] D. Topic, K. Malari ' c, and D. Muha, "Behavior of $2.4 \mathrm{GHz}$ unmanned aerial vehicle (UAV) under intereference," in Proceedings of the 2018 First International Colloquium On Smart Grid Metrology, pp. 1-4, Split, Croatia, April 2018.

[19] S. R. Pandey, K. Kim, M. Alsenwi, Y. K. Tun, and C. S. Hong, "A crowd-enabled task execution approach in UAV networks towards fog computing," in Proceedings of the 2021 IEEE International Conference On Big Data and Smart Computing, pp. 246-251, Jeju Island, Korea, 2021.

[20] L. Zhu, Y. Li, and F. R. Yu, "Cross-layer defense methods for jamming-resistant CBTC systems," in Proceedings of the IEEE Transactions On Intelligent Transportation Systems, pp. 1-13, Jeju Island, Korea, 2020.

[21] ECC, "A comparison of the minimum coupling loss method, enhanced minimum coupling loss method and the MonteCarlo simulation," CEPT, Menton, France, ERC Reports, vol. 101, 1999.

[22] W.-G. Chung, H.-S. Jo, H.-G. Yoon, J.-W. Lim, J.-G. Yook, and H.-K. Park, "Advanced MCL method for sharing analysis of IMT-advanced systems," Electronics Letters, vol. 42, no. 21, pp. 1234-1235, 2006.

[23] H.-S. Jo, H.-G. Yoon, J. Lim, and J.-G. Yook, “An advanced MCL method for assessing interference potential of OFDMbased systems beyond 3G with dynamic power allocation," in Proceedings of European Wireless, pp. 39-42, Manchester, U.K, September 2006.

[24] W. A. Hassan, H.-S. Jo, S. Ikki, and M. Nekovee, "Spectrumsharing method for Co-existence between 5G OFDM-based 
system and fixed service," IEEE Access, vol. 7, pp. 7746077475, 2019.

[25] Y. S. Cho, J. Kim, W. Y. Yang, and C.-G. Kang, MIMO-OFDM Wireless Communications with MATLAB, Wiley (Asia) Pte. Ltd, Singapore, 2010.

[26] Maximum permissible levels of off-axis e.i.r.p. density from earth stations in geostationary-satellite orbit networks operating in the fixed-satellite service transmitting in the $6 \mathrm{GHz}$, $13 \mathrm{GHz}, 14 \mathrm{GHz}$ and $30 \mathrm{GHz}$ frequency bands, ITU-R S.524-9, 2006.

[27] Calculation of free-space attenuation, ITU-R P.525-4, 2019.

[28] Feasibility of MSS operations in certain frequency bands, ITU-R M.2221, 2011.

[29] Attenuation by atmospheric gases and related effects, ITU-R P.676, 2019.

[30] Frequency and distance separations, document Rec, ITU-R SM.337-6, 2008. 